\title{
DISCURSO DE AULA Y ARGUMENTACIÓN EN LA CLASE DE CIENCIAS: CUESTIONES TEÓRICAS Y METODOLÓGICAS
}

\author{
Jiménez Aleixandre, María Pilar y Díaz de Bustamante, Joaquín \\ Departamento de Didáctica das Ciencias Experimentais \\ Universidade de Santiago de Compostela
}

\begin{abstract}
Resumen. Este trabajo constituye una reflexión sobre la investigación del discurso de aula y su contribución al conocimiento de los procesos de aprendizaje de las ciencias. Se trata con más detalle una parte de estos estudios, los que analizan el razonamiento argumentativo del alumnado, con ejemplos tomados del proyecto RODA (razonamiento, discusión, argumentación), llevado a cabo en la Universidad de Santiago de Compostela desde 1994, y se presentan otras dimensiones del discurso de aula estudiadas en el proyecto, como las operaciones epistémicas y la cultura escolar. Se discuten algunas implicaciones para la investigación en didáctica de ciencias.

Palabras clave. Discurso de aula, argumentación, comunicación, métodos de análisis.
\end{abstract}

Summary. This paper reviews the research line on classroom discourse and its contribution to the understanding of the science learning processes. The particular focus are the studies analysing the argumentative reasoning of students, discussing instances from the RODA project (Reasoning, Debate, Argumentation), carried out in the University of Santiago de Compostela since 1994. Other dimensions of the classroom discourse studied in the project such as epistemic operations and school culture are also discussed. Some implications for research in science education are outlined.

Keywords. Classroom discourse, argumentation, communication, methods of analysis.

\section{INTRODUCCIÓN}

El propósito de este artículo es discutir algunas cuestiones, tanto teóricas como metodológicas, sobre la línea de investigación del discurso de aula y la forma en que contribuye a una mejor comprensión de los procesos de aprendizaje de las ciencias. Al ser una línea reciente parece importante revisar tanto el marco teórico como algunos aspectos de los métodos utilizados en estas investigaciones. En el artículo se presta particular atención a una parte de estos estudios, los que analizan el razonamiento argumentativo del alumnado. La reflexión, tanto sobre la fundamentación teórica como sobre la metodología de investigación, se ilustra con ejemplos de resultados del proyecto RODA (razonamiento, discusión, argumentación), llevado a cabo en la Universidad de Santiago de Compostela desde 1994. El artículo, por tanto, presenta una estructura en la que se combinan tanto reflexiones sobre aspectos de la fundamentación teórica y la metodología seguida como datos de nuestra investigación en la mayoría de los cinco apartados:

- En primer lugar se presenta la línea de trabajo sobre el discurso de aula, enmarcándola en el análisis de la comunicación en las clases de ciencias, y se discuten sus relaciones con otras líneas de trabajo relevantes en las últimas décadas.

- El segundo apartado trata de cuestiones relacionadas con el marco teórico, lo que se entiende por argumentación y su relación con el razonamiento, particularmente en situaciones de construcción de nuevos conocimientos.

- En el tercero se discuten sobre todo cuestiones metodológicas suscitadas por el análisis del discurso y la argumentación, como el papel de los enunciados en el discurso. 
- El cuarto trata de otras dimensiones del discurso de aula, como las operaciones epistémicas y la cultura escolar. En los procesos comunicativos, todas estas dimensiones se presentan conectadas, aunque a efectos de análisis distingamos entre unas y otras.

- En último lugar, se discuten algunas implicaciones para la investigación en didáctica de ciencias, dificultades que plantean estos estudios y retos para el futuro.

\section{DISCURSO DE AULA: ANÁLISIS DE LA CO- MUNICACIÓN EN CLASE}

Durante la última década, diversos equipos de investigación en didáctica de ciencias se han interesado por el sistema de comunicación en las clases, por el discurso del aula. Debemos a Courtney Cazden (1991) la definición de este discurso como un sistema de comunicación así como un reconocimiento del papel que el lenguaje hablado juega en la enseñanza y el aprendizaje. Otros autores, como Sutton $(1992,1997)$ también han subrayado el importante papel jugado por la comunicación y el lenguaje en la construcción del conocimiento científico $\mathrm{y}$, en concreto, en el aprendizaje de las ciencias.

Según Cazden (1991), el discurso escolar es el sistema de comunicación implantado por el profesor o profesora; los significados que una clase y un docente concreto representan y realizan. Hay que tener en cuenta que el lenguaje hablado, la expresión oral, combina lo cognoscitivo y lo social (Vygotski, 1979) y, aunque algunos análisis se centran en uno solo de estos aspectos, no puede olvidarse la influencia que ejercen uno en otro. En el aula de ciencias, y en la enseñanza en general, la expresión oral es decisiva, entre otras razones, porque la instrucción procede, en gran medida, a través del lenguaje hablado y porque el aprendizaje se demuestra, en gran medida, a través del mismo.

En un aula, a diferencia de lo que ocurre en otros ambientes humanos, el control de lo que se habla «oficialmente» está en manos del profesor. Como hace notar Cazden, el sistema de comunicación escolar es un medio problemático, siendo el lenguaje hablado una parte importante de la identidad de quien lo usa. Según esta autora hay por lo menos tres lenguajes en el aula que se corresponden con distintas funciones lingüísticas: lenguaje del currículo, a través del que se realiza la enseñanza y se muestra lo aprendido; lenguaje de control, mantenido por el docente; y lenguaje de identidad personal, diferencias entre cómo y cuándo se dice algo.

Desde el punto de vista del aprendizaje de las ciencias, nos interesa el análisis de este sistema de comunicación para identificar procesos de aprendizaje u obstáculos al mismo. La comunicación en el aula debería permitir a los participantes construir significados compartidos (tanto en la dimensión cognitiva como en la social), lo que no siempre ocurre, pues, como indican Kelly y Crawford (1997), los estudiantes pueden compartir tareas o actividades sin compartir conocimiento, y por esta razón, en la práctica, distintos estudiantes de un mismo grupo tienen diferente acceso al conocimiento. El lenguaje científico no es unívoco, diferentes personas pueden asignar significados distintos a un mismo término y, como indica Sutton (1997), el lenguaje puede ser contemplado como un sistema de etiquetado o como un sistema de interpretación, perspectivas que tienen distintas implicaciones para la enseñanza de las ciencias. El análisis del discurso pretende profundizar en algunas de estas cuestiones relacionadas con el acceso al conocimiento.

Esta línea de investigación puede considerarse complementaria de otras que, durante las dos últimas décadas del siglo $\mathrm{XX}$, han explorado los conocimientos, ideas y representaciones del alumnado, sobre todo en cuanto al producto o resultado del aprendizaje, a la interacción entre las ideas previas de la persona que aprende y las estrategias o materiales de instrucción. En cierta medida, algunos trabajos sobre el cambio conceptual, como la tesis de Hennessey (1991), por su metodología de estudio de clase, pueden considerarse análisis del discurso del aula. Las relaciones y diferencias entre las investigaciones de ideas previas y de discurso de aula exceden el marco de este trabajo, pero se puede señalar que, mientras que muchos de los estudios sobre ideas previas se han realizado sobre muestras amplias a las que se han pasado pruebas escritas o entrevistas en momentos determinados, los de discurso de aula suelen ser estudios de caso sobre muestras reducidas y los datos se toman de forma continuada durante períodos relativamente largos, al menos una unidad didáctica o secuencia instruccional completa. Como se indicaba más arriba, estas diferencias metodológicas tienen que ver con el diferente objeto de estudio, en el primer caso, más centrado en productos y, en el caso de los estudios de discurso de aula, más centrado en procesos. Además de las conexiones con el cambio conceptual hay que señalar que una de las investigadoras más destacadas sobre ideas de los alumnos, Rosalind Driver, inició a finales de los noventa estudios sobre argumentación (Driver, Newton y Osborne, 2000), truncadas por su prematura muerte. En otras palabras, no cabe contemplar estas dos líneas como opuestas, sino como complementarias, explorando diferentes aspectos de los mismos problemas.

Por su carácter de estudios de caso, las investigaciones sobre discurso del aula son adecuadas para la investigación-acción, estudios en los que los docentes analizan su propia práctica en el aula, actuando como profesoresinvestigadores. Los docentes dispuestos a emprender esa reflexión son, en general, personas implicadas en la innovación educativa, y sus clases y estrategias suelen presentar diferencias con la mayoría, lo que lleva a plantearse la cuestión acerca de la validez y la representatividad de estos estudios. ¿Es útil estudiar lo que ocurre en clases innovadoras? ¿No serán poco representativas? Ann Brown (1992), implicada en el proyecto que pretendía transformar las clases en comunidades de aprendizaje, respondió a estas preguntas sugiriendo que estos estudios de aula eran más interesantes, en este momento, que los estudios de aprendizaje en condiciones de laboratorio (entrevistas clínicas u otros realizados fuera del contexto real de una clase de ciencias), ya que, al ser las clases sistemas muy complejos, no admiten bien el aislamiento de uno de sus componentes para ser estudiado de forma independiente. 
Según Brown, uno de los desafíos que se plantean a la investigación educativa es precisamente el diseño de unidades y estrategias innovadoras y su evaluación mediante estudios de caso. En cuanto a la singularidad de las clases innovadoras y el riesgo de que en estas investigaciones se produzca el «efecto Hawthorne» (que la expectativa de mejores resultados influya en su obtención), Brown afirma que esos buenos resultados son el objetivo de la enseñanza. Como se discute en otro trabajo (Jiménez y López, 2001), creemos que la proliferación de estudios en los que se muestra sobre todo lo poco que aprende el alumnado, los problemas de aprendizaje de conceptos o lo inadecuadas que son las estrategias de los docentes, por ejemplo, para desarrollar una verdadera educación ambiental, pueden llevar al desánimo al profesorado y también pensamos que son necesarios estudios que muestren la existencia de buenas prácticas, de clases donde los alumnos y alumnas se interesan por problemas relacionados con las ciencias o el ambiente y trabajan como una verdadera comunidad de aprendizaje.

En esta perspectiva, los objetivos de la enseñanza de las ciencias, como el aprendizaje de conceptos y modelos o el desarrollo de actitudes y destrezas, forman parte de la adopción por parte del alumnado de la cultura científica, transformando la clase de ciencias en una comunidad donde se produce y se usa el conocimiento. Esta perspectiva de la clase como comunidad de aprendizaje (McGinn y Roth, 1999) debe prestar atención al diseño de un contexto en el que el alumnado se implique en la resolución de problemas auténticos, en tareas que sean relevantes para su vida, pues en las aulas en que su papel es pasivo o poco activo apenas hay comunicación de los estudiantes entre sí.

Entre los constructos y metodologías utilizados en el análisis, algunos proceden no sólo de la psicología educativa, sino también de la antropología, como la cultura de un campo (la cultura científica o la cultura escolar), en el sentido acuñado por Clifford Geertz (1973), como conjunto de símbolos significativos que la gente usa para hacer inteligibles sus vidas. Como indica Geertz, el investigador puede interpretar estos datos (metodologías interpretativas), pero no deducir o inferir de ellos relaciones causales en la forma en que se haría en ciencias experimentales.

A continuación se discute con más detalle una parte de esta línea, la que analiza el razonamiento argumentativo del alumnado.

\section{ARGUMENTACIÓN Y RAZONAMIENTO: CO- NOCIMIENTO ESTABLECIDO, CONOCIMIEN- TO NUEVO}

Dentro de las múltiples señales emitidas en las clases de ciencias, de los diferentes aspectos de la comunicación (por ejemplo, ideas del alumnado, modelos utilizados, interacciones en los pequeños grupos), en este trabajo se presta especial atención a los que tienen que ver con el razonamiento y la argumentación.
La perspectiva que contempla el aprendizaje de las ciencias como argumentación, y no sólo como exploración, ha sido propuesta por Deanna Kuhn (1992, 1993) y elaborada por otros autores como Driver y otros (2000) y Duschl (1997). Por argumentación se entiende la capacidad de relacionar datos y conclusiones, de evaluar enunciados teóricos a la luz de los datos empíricos o procedentes de otras fuentes. El razonamiento argumentativo es relevante para la enseñanza de las ciencias, ya que uno de los fines de la investigación científica es la generación y justificación de enunciados y acciones encaminados a la comprensión de la naturaleza (Jiménez, Bugallo y Duschl, 2000), por lo que la enseñanza de las ciencias debería dar la oportunidad de desarrollar, entre otras, la capacidad de razonar y argumentar (Jiménez, 1998; Sardá y Sanmartí, 2000). Para poder construir modelos, explicaciones del mundo natural y operar con ellos, las y los estudiantes necesitan, además de aprender significativamente los conceptos implicados, desarrollar la capacidad de escoger entre distintas opciones o explicaciones y de razonar los criterios que permiten evaluarlas (Zohar y Nemet, 2002). Los estudios de Kuhn (1992) indican que este desarrollo de destrezas argumentativas no tiene lugar en todos los contextos escolares y esta autora propone que la capacidad de emitir juicios razonados sea considerada parte de «pensar bien», ya que el conocimiento puede ser contemplado como un proceso permanente de evaluación, en que la modificación de las conclusiones se debe a la aparición de nuevos datos y nuevos argumentos, y no (añadiríamos nosotros) a un mero cambio de opinión.

La cuestión es: ¿podemos conocer el razonamiento argumentativo del alumnado, los procesos que tienen lugar en su mente? En opinión de Kuhn, el diálogo argumentativo exterioriza el razonamiento argumentativo. Es decir, no hay forma de conocer exactamente lo que ocurre en el interior de la mente, pero una de las formas en que podemos aproximarnos es prestando atención a las discusiones entre estudiantes sobre cuestiones de ciencias. Por esa razón gran parte de los estudios sobre la argumentación del alumnado, por ejemplo, los de Gregory Kelly y colaboradores (Kelly, Drucker y Chen, 1998), el proyecto RODA de la USC y el que se lleva a cabo en el King's College (Duschl, Ellenbogen y Erduran, 1999) se centran en el discurso natural que tiene lugar en las clases de ciencias, más que en respuestas obtenidas mediante cuestionarios o entrevistas.

Es importante discutir con cierto detalle la relación entre argumentación y razonamiento. El papel de la lógica en la argumentación ha sido muy discutido y, aunque algunos autores ven la argumentación sólo como una de las formas del razonamiento lógico, nosotros adoptamos la posición de Hintikka (1999), quien establece una diferencia entre, por un lado, la concepción tradicional de la lógica formal y, por otro, la lógica y la argumentación en el discurso natural, pues, siguiendo a este autor, para los teóricos del razonamiento humano, «las verdades de la lógica formal son meras tautologías o verdades analíticas sin contenido substancial y, por tanto, incapaces de apoyar ninguna inferencia que conduzca a descubrimientos nuevos o al menos sorprendentes» (Hintikka, 1999, p. 25). 
En nuestra opinión (Díaz y Jiménez, 2001), esto significa que, mientras que la lógica formal puede ser usada para representar o analizar el conocimiento establecido, no es un marco adecuado para interpretar el discurso en las situaciones en que se está generando conocimiento nиеvo. En el discurso natural, en este tipo de situaciones, por ejemplo, cuando se está resolviendo un problema en la clase de ciencias o en el laboratorio, pueden formularse enunciados que no sean totalmente correctos o incluso que sean falacias desde la perspectiva de la lógica formal, pero que al mismo tiempo constituyen pasos fructíferos en la construcción del conocimiento. Consideremos, por ejemplo, el siguiente fragmento de transcripción en el que unos alumnos de $3^{\circ}$ de ESO (15 años) están intentando identificar una muestra desconocida en una preparación microscópica, averiguar si es animal o vegetal y si tiene más de un tipo de células (Apéndice):

114 Flavio: Esto no tiene estomas.

115 Fabri: Pero tenía el coso verde. ¡Eh! Lo que... yo creo es clorofila.

El argumento de Fabri puede cumplir su función en el diálogo, persuadir a los otros miembros del equipo de que la solución propuesta - una muestra vegetal- es la correcta, a pesar de que una de las premisas sea falsa o al menos incierta, pues el hecho de que alguna estructura celular se vea de color verde a través del microscopio no significa necesariamente que contenga clorofila, sino que puede deberse a una tinción. Esto invalidaría el argumento desde el punto de vista de la lógica formal, pero en el proceso de argumentación de estos estudiantes, que más adelante se hace muy sofisticado hasta llegar a concluir que se trata de tejido conductor vegetal, ha constituido un paso adelante.

Esto puede expresarse también diciendo que el diálogo argumentativo se inscribe en la perspectiva de la lógica informal (Walton, 1989). Según Walton, el análisis del discurso argumentativo sobre cuestiones polémicas en lenguaje natural requiere, entre otras cosas, prestar atención al lenguaje, ser capaz de analizar proposiciones relativamente ambiguas o vagas, y además las personas que investigan este tipo de discurso deben estar preparadas para desenredar la línea fundamental de argumentación en medio de extensos intercambios entre dos o más personas. Walton hace notar que, en esta perspectiva dialéctica, se pone de manifiesto el contexto de pregunta /respuesta de algunos argumentos, contemplando estos como parte de un diálogo interactivo entre dos o más personas razonando juntas, lo que nosotros denominamos «co-construcción» de argumentos.

Se pueden resumir estas posturas diciendo que la argumentación en el contexto educativo, del aprendizaje de las ciencias, que tiene una dosis de ambigüedad, no se rige exactamente por los mismos patrones que la argumentación en filosofía, que sigue las reglas de la lógica, los cuales demandan abstracción y precisión.

También Driver y otros (2000) contemplan la argumentación como una práctica humana, sea individual o social, regida por mecanismos diferentes a las reglas abstractas de la lógica. Estos autores distinguen entre diferentes tipos de argumentos:
- Retóricos, razones para convencer al auditorio, frente a dialógicos, que examinan distintas alternativas, siendo éstos los de mayor interés para el análisis del discurso.

- Racionales, que buscan una solución racional a un problema determinado, frente a persuasivos, que pretenden llegar a un consenso.

Como señalan Driver y otros (2000), todos los argumentos están situados, es decir, influidos por una cultura, época e ideología determinadas, pero coincidimos con estos autores en que esto no implica que sean totalmente relativos, ya que, en muchos casos, existen criterios para comparar enunciados alternativos y escoger el más adecuado.

La siguiente cuestión que se plantea es cómo investigar esta parte del discurso del aula, qué estudiar en cuanto a la argumentación en las clases de ciencias, y cómo estudiarlo, qué métodos utilizar, lo cual es objeto del siguiente apartado.

\section{NATURALEZA DE LOS ENUNCIADOS Y FUN- CIÓN EN EL DISCURSO}

Parece existir acuerdo entre distintos autores sobre la importancia de que el alumnado desarrolle su capacidad de argumentar y, para poder diseñar estrategias que incluyan este objetivo, es importante analizar cómo lo hacen. Como indican Ogborn, Kress, Martins y McGillicuddy (1998), es necesario comprender cómo se construyen las explicaciones en el aula, explorar los procesos a través de los cuales se van construyendo significados en las clases de ciencias, qué significado asignan los alumnos y alumnas a entidades (que pueden ser abstractas) como gen, célula, fuerza o neutralización.

Algunas de las preguntas que nos hacemos al estudiar la argumentación son:

- ¿Qué cuenta como dato, como comprobación para las y los estudiantes?

- ¿Qué cuenta como conclusión o explicación teórica?

- ¿Qué cuenta como justificación en apoyo de una conclusión? ¿En qué situaciones utilizan justificaciones los estudiantes? ¿Qué tipos de justificaciones?

- ¿Cómo se relacionan las justificaciones con el conocimiento básico que las respalda?

- ¿Qué proceso siguen para argumentar mientras están resolviendo un problema?

Cuando nos preguntamos qué cuenta como dato, qué cuenta como conclusión, qué como justificación, a lo que nos referimos es a qué vale como dato, justificación, etc. para el alumnado, cómo los emplea. El análisis del discurso es un método que tiene un componente subjetivo y en el que distintos investigadores pueden interpretar de distinta forma un diálogo o acción. Los debates sobre cómo interpretar fragmentos específicos del discurso en el aula, sobre si un enunciado puede 
considerarse dato o justificación (u otro elemento) nos llevan a establecer una distinción entre la naturaleza de los enunciados, es decir, lo que son desde el punto de vista ontológico y su papel en el discurso, como elementos de la argumentación. Por ejemplo: cuando una persona dice que determinada muestra vista al microscopio es un vegetal porque se ve de color verde, lo que indica presencia de clorofila, el color verde de la muestra es un dato empírico, desde el punto de vista ontológico, mientras que su papel en el discurso es de justificación que sustenta la conclusión: es una muestra de tejido vegetal. Creemos que ésta es una importante distinción que debe ser tenida en cuenta a la hora de realizar un análisis. A continuación se reproduce un fragmento de transcripción de otro grupo de alumnado (en una mezcla de gallego y castellano) con la misma tarea de identificación de una muestra desconocida en el microscopio:

23 Emilio: Esto se parecen como... como... como as dendritas as cousas esas, ¡tío!

24 Eloy: ¿Cómo?

25 Emilio: Como... dendritas, como... los rollos de ... ¿Cómo se llama eso... en la cabeza? Dendritas, eh... neuronas. ¿No te fijas que ten os rollos ramificaos?

180 Zenón: ¡Esto es animal, eh!

181 Emma: ¿Es animal?

182 Zenón: Sí.

183 Emma: ¿Por qué?

184 Zenón: Porque aparecen neuronas por ahí... ¡Yo que sé!

En las primeras líneas, Emilio está intentando buscar el término adecuado para describir sus observaciones: células que interpreta como neuronas. Aun cuando en realidad se trata de osteoblastos en opérculo de pez, el objetivo no era que identificasen exactamente la muestra, sino que reconocieran el tipo (animal o vegetal) y dentro de las animales, con uno o dos tipos distintos de células. En las últimas líneas, la observación de estas «neuronas», que constituye un dato empírico, es utilizado por Zenón como justificación de su conclusión: se trata de un tejido animal.

Las cuestiones metodológicas que plantea el análisis del discurso son complejas y van desde cómo organizar los datos, qué instrumentos utilizar para interpretarlos, hasta cómo presentarlos y representarlos por medio de inscripciones. Para ello se utilizan métodos y constructos que proceden de otras ciencias sociales, como la etnografía y la antropología. Así se ha empleado lo que en antropología se conoce como trabajo de campo u observación participante, es decir, participación de una persona, registrando los acontecimientos que tienen lugar en las clases. También se tienen en cuenta estudios de sociología de la ciencia; así, los que analizan los procesos discursivos en laboratorios científicos. Latour y Woolgar (1995) estudian la construcción de hechos, la transformación de datos a través de las conversaciones -discurso-, los procesos por los que los científicos y científicas dan significado a sus observaciones. Así, han documentado ejemplos de transformación de enunciados en hechos o lo que describen como esfuerzos por introducir orden en un conjunto desordenado de observaciones, y contemplan las inscripciones y la acción de escribir como operaciones materiales encaminadas a crear orden, más que a la transferencia de información.
La transformación de un enunciado, que en un primer momento puede tener carácter individual e hipotético, en un «hecho» aceptado por la comunidad científica, implica, como señala Sutton (1997), un cambio tanto en el lenguaje como en el estatus del conocimiento. Un ejemplo (Jiménez Aleixandre, 2003) puede ser el caso de los priones, partículas proteínicas agentes de la EEB (enfermedad de las «vacas locas»):

1) Enunciado con estatus provisional, dudoso: «Prusiner propone que los agentes que causan estos procesos degenerativos del sistema nervioso podrían ser proteínas.»

2) Hecho aceptado: «Prusiner ha descubierto un nuevo tipo de agentes infecciosos, los priones.»

3) Conocimiento implícito: «Los priones están presentes en el tejido nervioso de los animales afectados.»

Esta perspectiva sociológica ha sido aplicada al estudio de las clases y los laboratorios por el equipo de Kelly, en el estudio de la argumentación de estudiantes de secundaria en el laboratorio (Kelly y Crawford, 1997; Kelly, Drucker y Chen, 1998) o de sus demandas comunicativas mientras diseñan y realizan montajes (Kelly y Brown, 2000), es decir, para explorar lo que cuenta (es considerado) como ciencia en las clases. Los estudios de Kelly y sus colegas son ejemplos de una nueva forma de explorar el pensamiento de los estudiantes a través del estudio directo del discurso, más que a través del análisis de respuestas a cuestionarios.

En nuestra opinión, el análisis del discurso a través de las transcripciones y registros de clase puede compararse a estudiar una muestra con un microscopio: se necesitan diferentes enfoques con distintos aumentos para llegar a tener una idea adecuada de la preparación. Algunas de las representaciones que utilizan en el proyecto RODA, para comprender lo que ocurre en las clases, son, de mayor a menor detalle:

1) Secuencias de actividades o fases en una unidad didáctica, por ejemplo la de la figura 1, tomada de López Rodríguez (2001).

2) Secuencias de episodios, por ejemplo, en cada sesión de una unidad (Jiménez y López, 2001) y ejes cronológicos (timelines), en Reigosa, Jiménez y García Rodeja (2001).

3) Secuencias de argumentos a lo largo de una o varias sesiones de trabajo, como las estudiadas durante la identificación de muestras desconocidas con el microscopio (Díaz de Bustamante, 1999) una de las cuales se reproduce en la figura 2.

Cabe señalar que nuestro análisis de la argumentación no se realiza sobre cada frase o grupo de frases que puede ser considerado un argumento desde el punto de vista formal, sino únicamente sobre aquéllos que Toulmin (1958) denomina argumentos substantivos, que requieren un conocimiento del contenido. En el proyecto RODA algunos de los pasos seguidos son:

- Identificar elementos o componentes de los argumentos, siguiendo el esquema propuesto por Toulmin (1958). 
Figura 1

Secuencia de actividades en la unidad (López Rodríguez, 2001).

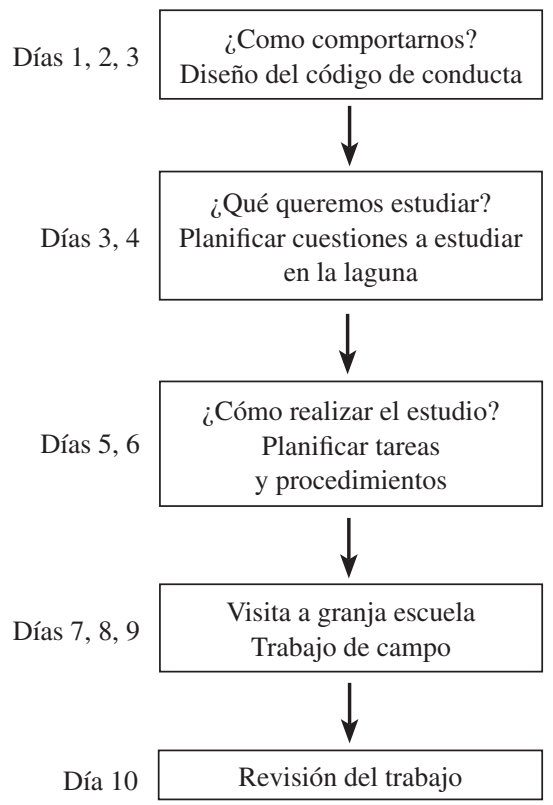

Figura 2

Secuencia de argumentos, $3^{\circ}$ de ESO (Díaz de Bustamante, 1999).

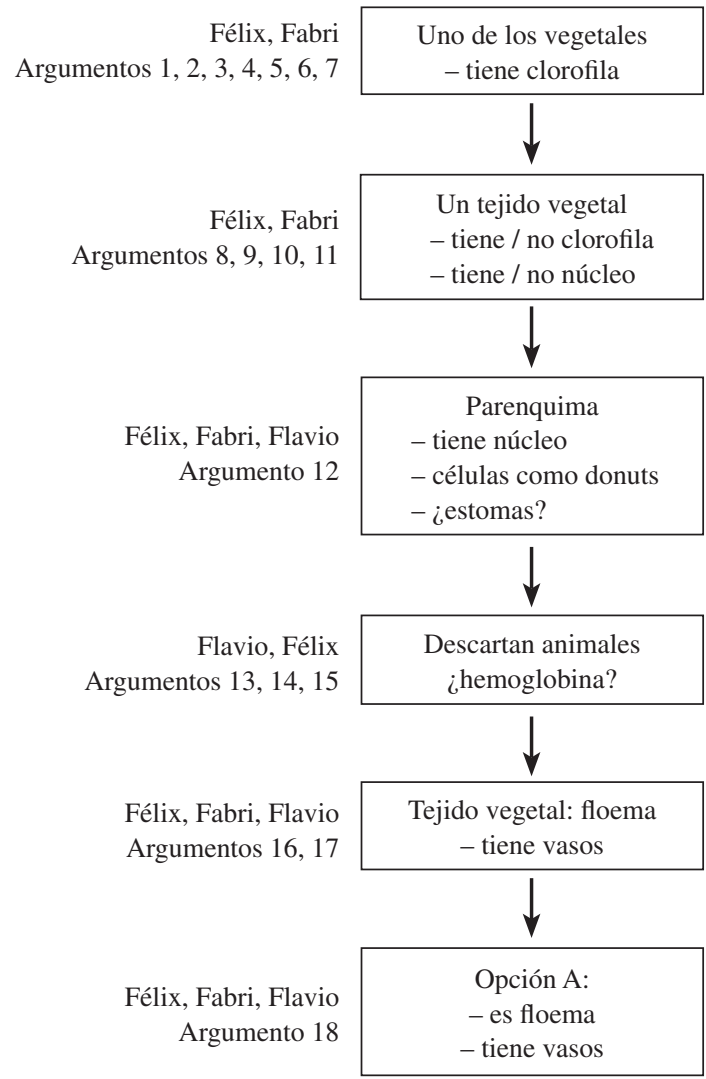

- Identificar, por ejemplo, en las transcripciones de un pequeño grupo, los argumentos substantivos cuya conclusión, explícita o implícita, constituye una respuesta al problema planteado, como sería una hipótesis respecto a la muestra (animal, vegetal, tipo de tejido) en la tarea del microscopio, una evaluación del proyecto de gestión ambiental (Jiménez, Pereiro y Aznar, 2000), una propuesta sobre cómo realizar una valoración ácido/base (Reigosa y Jiménez, 2001). Un fragmento de diálogos y discusiones, cualquiera que sea su longitud, se considera parte del mismo argumento cuando la conclusión es la misma, y distinto cuando se modifica. A partir de ahí se puede elaborar la secuencia de argumentos para esa sesión y grupo. La representación de estas secuencias, como la que aparece en la figura 2, ayuda a hacer visible el proceso por el que un argumento cambia, o cómo se usan diferentes justificaciones para el mismo. Así, en la figura 2 puede observarse cómo parte de las discusiones giran en torno a la cuestión de si la muestra es de tejido animal o vegetal, mientras que en otras se trata de identificar qué tejido es (aunque, como hemos indicado más arriba, esta identificación no se solicitaba; este grupo de $3^{\circ}$ de ESO, llegó a la conclusión, correcta, de que en la muestra había floema).

- A continuación puede procederse a analizar la calidad de los argumentos: consideramos de mayor calidad los justificados. Un análisis posterior puede establecer di- ferentes tipos de justificaciones y condiciones para las mismas (Kelly et al., 1998; Díaz y Jiménez, 2001).

4) Representaciones de la heurística o pasos en la resolución de un problema, por ejemplo, la que aparece en la figura 3, tomada de Reigosa Castro (2002).

5) Pasos en la transformación de las propuestas y construcción de datos por los estudiantes, por ejemplo, la que aparece en la figura 4, de Díaz de Bustamante (1999).

Estas representaciones, a distintas escalas, permiten estudiar la argumentación del alumnado, no sólo desde el punto de vista de cada argumento, sino también del proceso de construcción, «co-construcción», modificación y cambio que los argumentos sufren.

Un potente instrumento para el análisis de la argumentación es el modelo de Stephen Toulmin (1958), que establece una serie de componentes y sus relaciones en la conversación natural. Estos componentes, que se discuten con más detalle en Jiménez (1998), son: a) datos, en los que, siguiendo en parte a Kelly y otros (1998), distinguen entre datos suministrados y obtenidos, y dentro de éstos, entre empíricos (por ejemplo, de una experiencia de laboratorio) e hipotéticos; b) enunciados, dis- 
tinguiendo entre hipótesis y conclusiones; $c$ ) justificaciones específicas para la argumentación; d) conocimiento básico, de carácter general. A estos cuatro se agregan, en algunos casos: e) calificadores modales; y f) refutación. Estos componentes pueden estar explícitos o implícitos. En la figura 5 aparece un ejemplo de argumento (Díaz y Jiménez, 2001) en el formato de Toulmin. Las dos alumnas que componen el grupo habían mantenido, unos turnos más arriba, que en la muestra había dos tipos distintos de células; sin embargo, en este momento cambian de criterio:

140 Gloria: Pues claro que son todas del mismo tipo. Unas son más grandes y otras más pequeñas, pero todas del mismo tipo.

Gloria interpreta ahora la observación, no como la presencia de distintos tipos celulares, sino como una diferencia de tamaño, lo que, en nuestra opinión, constituye un ejemplo de construcción de datos, en el sentido de transformar una descripción a través de sucesivas interpretaciones. Se representa como un argumento en el que una justificación se apoya en otra.

Figura 3

Heurística (Reigosa, 2002).

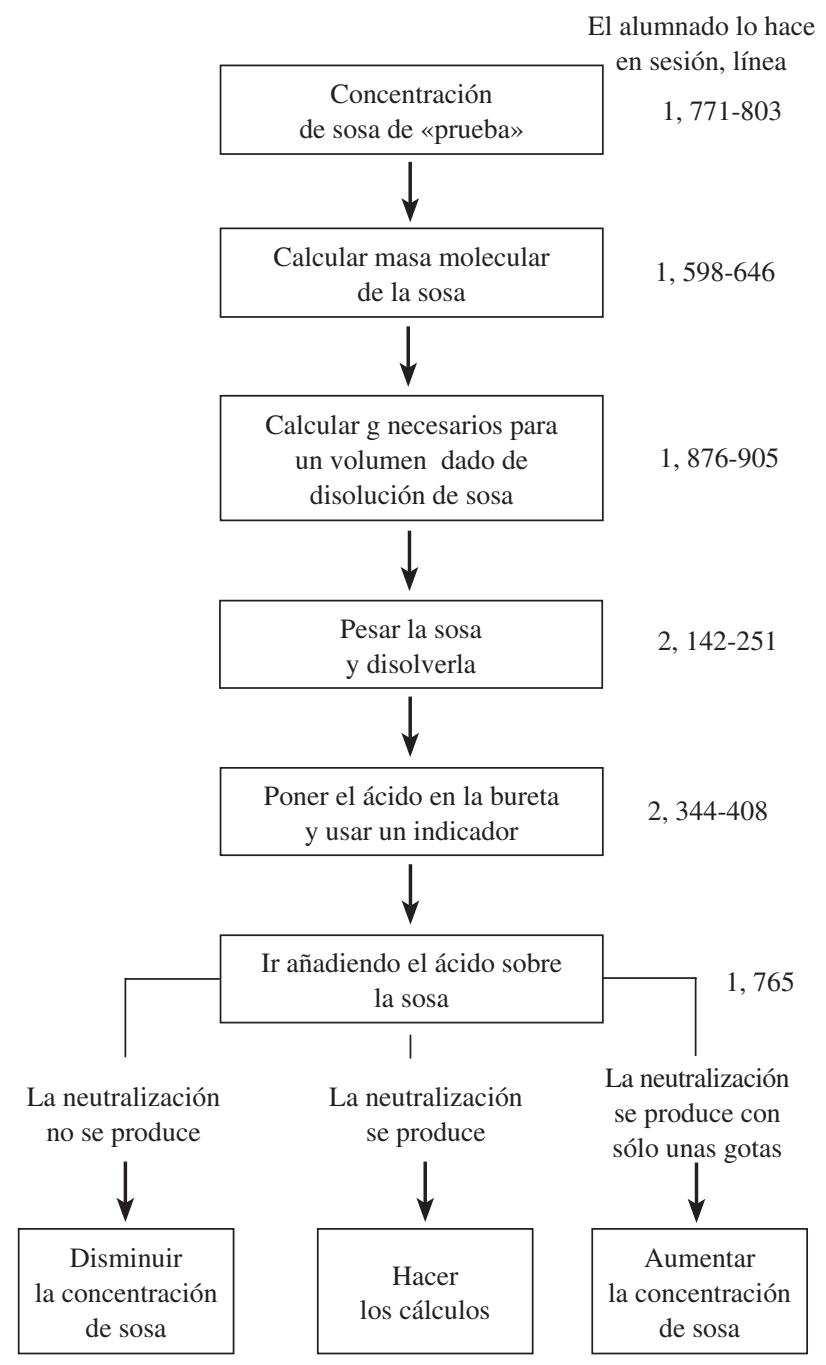

El esquema de Walton (1996) es visto por Duschl y otros (1999) como particularmente adecuado para la estructura dialéctica y la secuencia de razonamiento argumentativo del diálogo grupal. Las categorías de Walton, en particular lo que denomina argumento basado en la opinión de expertos, ha sido utilizado (Jiménez, Pereiro y Aznar, 2000) para construir un esquema de análisis del razonamiento del alumnado en cuanto al estatus de experto (quién lo tiene, qué consistencia hay en sus enunciados, etc.).

Este breve resumen puede dar una idea de algunas de las cuestiones metodológicas con las que nos enfrentamos al analizar la argumentación del alumnado, aunque también hay dificultades no del todo resueltas y nuevas perspectivas de investigación que se discuten en el apartado de implicaciones. A continuación, como muestra de la complejidad de los procesos de comunicación en las clases de ciencias, se tratan brevemente otras operaciones identificadas en el discurso del aula.

Figura 4

Transformación de datos (Díaz de Bustamante, 1999).
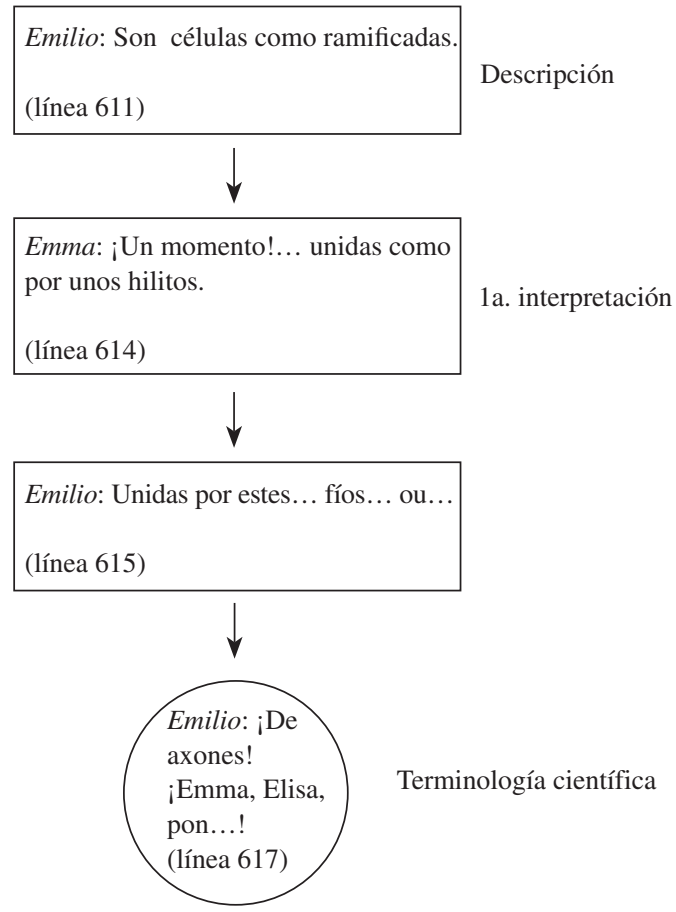
Figura 5

Argumento en formato Toulmin, grupo G (Díaz y Jiménez, 2001).

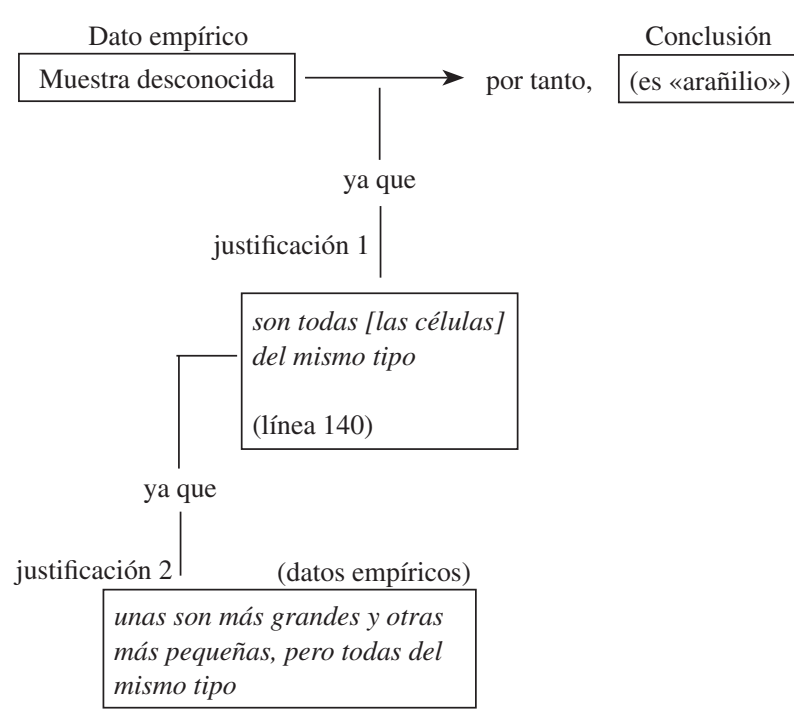

\section{OPERACIONES EPISTÉMICAS, PRÁCTICA CIENTÍFICA: OTRAS DIMENSIONES DEL DIS- CURSO}

Determinados enunciados y acciones del alumnado, aunque pueden ser analizados desde el punto de vista de la argumentación, muestran también la existencia de operaciones de construcción del conocimiento, operaciones epistémicas. Además en los análisis de sesiones de laboratorio, creemos que hay que prestar atención a las operaciones procedimentales y técnicas; siendo ambos tipos de operaciones característicos de la práctica científica. El análisis del discurso de los estudiantes está guiado, por una parte, por las decisiones epistémicas acerca de «qué cuenta como» (dato, observación, comparación con prototipo, etc.), que tienen lugar durante las transformaciones de datos y, por otra parte, por las operaciones procedimentales y técnicas durante la adquisición de datos. Hay que tener en cuenta que, aunque se construyan categorías para analizarlas por separado, muchas veces se solapan.

Operaciones epistémicas son, por ejemplo, los procedimientos explicativos, definiciones, clasificaciones, relaciones causales, apelaciones a analogías o comparaciones, o construcción de datos, que pueden ser interpretadas como características de los procesos de construcción del conocimiento en ciencias experimentales. Pontecorvo y Girardet (1993) han propuesto un esquema para interpretar las operaciones epistémicas en ciencias sociales y Jiménez, Díaz y Duschl (1998), para ciencias experimentales incluyendo tanto las operaciones epistémicas como las procedimentales y técnicas para el caso del uso del microscopio en el laboratorio de biología.

Las operaciones procedimentales y técnicas incluyen no sólo el uso de instrumentos y aparatos específicos (por ejemplo, en el caso del microscopio, realizar la prepa- ración, enfocar, cambiar de objetivo, etc.), sino también las discusiones y propuestas sobre este uso, que prueban la comprensión (o no) del propósito de la operación física, así como categorías más generales, como uso de inscripciones. Un aspecto de particular interés es la conciencia de los límites que el instrumento impone a la observación (en el caso del microscopio) o medición, de la interacción entre el instrumento y el mundo material, los artefactos y sesgos que puede crear.

Un modelo de análisis de las operaciones epistémicas, que resulta particularmente interesante para obtener una perspectiva de conjunto del progreso del alumnado, es el de Duschl y Erduran (1996), que establece una secuencia de pasos en el proceso de transformación, desde los datos en bruto a las explicaciones teóricas o teorías científicas. En la tabla I hemos representado esta secuencia para la tarea de identificar una muestra al microscopio.

En el primer paso hay una selección o construcción de datos, cuando del conjunto de datos disponibles se eligen sólo unos cuantos para ser objeto de atención. El segundo es la interpretación de los datos para identificar pautas y el tercero propone una explicación teórica que dé cuenta de las pautas. Según nuestros estudios, no todos ellos presentan el mismo grado de dificultad para las y los estudiantes.

Por último, cabe tener en cuenta que no todas las operaciones realizadas por los estudiantes, no todas sus justificaciones pueden situarse en el dominio de la cultura científica. Hay otras que se pueden situar de forma más apropiada bajo una categoría que recibe diferentes nombres: cultura escolar (como opuesta a la cultura científica); o, en términos de Habermas (1987), acciones dramatúrgicas, categoría utilizada en Reigosa y Jiménez (2000), en las que los participantes constituyen una audiencia mutua para un papel representado, y que este autor opone a las acciones comunicativas, orientadas a la comprensión mutua. Para estudiar esta dimensión, consideramos útil la idea de Bloome, Puro y Theodorou (1989) de «exhibición procedimental» (procedural display), que definen como la exhibición, por profesores y estudiantes, de una serie de procedimientos, académicos e interactivos, que cuentan como la realización de una lección, pero que no siempre están relacionados con los aprendizajes de contenidos o destrezas, sino más bien con los significados y valores culturales mantenidos por la comunidad respecto a la educación. Se ha utilizado esta idea para analizar las discusiones de estudiantes sobre un problema de genética (Jiménez, Bugallo y Duschl, 2000), oponiendo este «hacer clase» frente a «hacer ciencia»; es decir, diálogo científico y argumentación. Un ejemplo de acción dramatúrgica se encuentra durante la tarea de separar sal de arena propuesta a los estudiantes (Reigosa Castro, 2002). El grupo analizado toma una pequeña cantidad y diseña un procedimiento de separación, pero, a continuación, se dan cuenta de que los restantes equipos de la clase han separado la totalidad de la muestra y proceden a repetir los pasos con lo que les queda. En otras palabras, interpretamos que, a pesar de que habían resuelto el problema (pues la tarea solicitaba el diseño de un procedimiento, no la separación completa), consideran que, para cumplir con su «papel» de estudiantes, deben separar toda la mezcla. 
Tabla I

Secuencia de transformación de datos.

\begin{tabular}{|l|l|l|l|}
\hline \multicolumn{1}{|c|}{ Datos en bruto $\longrightarrow$} & \multicolumn{1}{|c|}{ Datos duros $\longrightarrow$} & \multicolumn{1}{c|}{ Pautas $\longrightarrow$} & \multicolumn{1}{c|}{ Explicaciones } \\
\hline $\begin{array}{l}\text { Observar la preparación y «ver» } \\
\text { las células }\end{array}$ & $\begin{array}{l}\text { Hallar la correspondencia entre } \\
\text { las células de la muestra y un } \\
\text { prototipo }\end{array}$ & $\begin{array}{l}\text { Por ejemplo: } \\
\text { Distinguir células individuales } \\
\text { de grupos de células }\end{array}$ & $\begin{array}{l}\text { Por ejemplo: } \\
\text { Identificar la muestra como célu- } \\
\text { las animales de un mismo tipo }\end{array}$ \\
\hline
\end{tabular}

\section{CAMINOS A SEGUIR: DIFICULTADES EN- CONTRADAS Y NUEVOS DESAFÍOS}

En este trabajo se discuten algunas de las cuestiones teóricas y metodológicas suscitadas por el estudio del discurso del aula, en el marco del proyecto RODA, llevado a cabo en la USC con el objetivo de estudiar los procesos de razonamiento y argumentación del alumnado de secundaria durante la resolución de problemas de ciencias y ambientales. En el marco del proyecto se han elaborado o adaptado diferentes herramientas que permiten profundizar en el análisis del complejo sistema de comunicación del aula. Existen múltiples dimensiones del discurso: razonamiento, argumentación en cuanto a la relación entre datos y conclusiones teóricas, compromisos epistemológicos, justificaciones de acciones y propuestas, valores. Se pretende que estos trabajos colaboren a la perspectiva de la clase como una comunidad de aprendizaje en la que el conocimiento es compartido por docentes y estudiantes, como una comunidad de pensamiento (Sutton, 1992).

Incluir la capacidad de argumentación en los objetivos de la enseñanza de las ciencias significa, entre otras cosas: reconocer las complejas interacciones que tienen lugar en el aprendizaje, así como la contribución de las prácticas discursivas en la construcción del conocimiento científico; tener en cuenta que hacer ciencia es también proponer y discutir ideas, evaluar alternativas, elegir entre diferentes explicaciones y ampliar la visión del aprendizaje de las ciencias. En resumen, puede decirse que su objetivo es la participación de las y los estudiantes en el discurso de las ciencias. En las clases que siguen una metodología tradicional, esta participación apenas existe, pues pocas veces se da a los estudiantes la oportunidad de resolver problemas o evaluar alternativas. Por ejemplo, observar y dibujar epitelio de cebolla en el microscopio no presenta una gran demanda cognitiva, ni requiere la resolución de un problema. Es importante diseñar actividades que ayuden al alumnado a «hablar ciencias», a participar de la cultura científica, de la producción y circulación de conocimiento.

Metodológicamente se recogen datos de diversas fuentes: grabaciones en audio y vídeo, productos del alumnado, carpetas o portafolios, empleando asimismo la observación participante. En unos casos se trata del propio docente, en la tradición del profesor-investigador; en otros es un observador u observadora que forma parte de la clase durante todo el período dedicado a una unidad. Al estudiar todo un sistema de comunicación, el análisis se realiza sobre unidades completas, durante uno o más meses.
En las tesis realizadas en el marco del proyecto se abordan diferentes aspectos del discurso, por ejemplo, la resolución de problemas en el contexto del laboratorio, sea de biología o de física y química. La transformación de las prácticas en verdaderos problemas (Jiménez, 1998) es necesaria si el trabajo de laboratorio ha de ser parte de la inmersión en la cultura científica y no una mera ilustración de la teoría. Para ello es importante no sólo la propia resolución del problema, sino la reflexión sobre el camino seguido, la justificación de las acciones o propuestas y especialmente el análisis de las dificultades que las tareas abiertas plantean al alumnado. También se explora la validez de las propias herramientas utilizadas en el análisis. Algunas de las investigaciones abordan cuestiones ambientales, en el marco de un estudio longitudinal a lo largo de tres cursos (López Rodríguez, 2001), que pretende documentar el desarrollo de la competencia del alumnado en este terreno analizando la compleja relación entre valores y actitudes, por un lado, y comportamiento, por otro, o, en palabras de uno de los alumnos de $6^{\circ}$ de primaria, «entre teoría y práctica». En otros casos, por ejemplo, en Pereiro (2001), se aborda la argumentación sobre gestión ambiental de seis grupos en $3^{\circ}$ de BUP, durante una unidad dedicada a evaluar un proyecto de saneamiento en un humedal, un problema no sólo auténtico, sino real. La comparación entre los argumentos, en el formato de Toulmin, de los distintos grupos y el análisis de sus justificaciones permite discutir aspectos como la relación entre conceptos y valores o las jerarquías establecidas entre estos últimos.

Los estudios de discurso de aula no están exentos de dificultades; algunas que nos parecen relevantes son:

\section{De carácter teórico:}

- El análisis, por su carácter interpretativo es una de las interpretaciones posibles, pudiendo haber otras.

- La combinación de diversos métodos y datos procedentes de distintas fuentes aumenta la fiabilidad (si los resultados son concurrentes), ya que no es posible trabajar con muestras amplias.

- Hay que tener en cuenta que determinados alumnos, y quizá especialmente alumnas, intervienen poco en los debates orales y en diseñar métodos para recoger datos de estas personas (que pueden tener razonamientos muy interesantes sin exponerlos en voz alta).

\section{De carácter metodológico:}

- Algunas elecciones difíciles: grabar un pequeño grupo o todos. 
- Obstáculos técnicos: dificultades para grabar la discusión entre varias personas.

- Dificultades para realizar estudios longitudinales en secundaria.

Por último, cabe señalar que hay nuevos desafíos, cosas que se pueden estudiar en este campo, por ejemplo: cómo se aprende a razonar, la dependencia (o no) entre la argumentación y el contenido, la continuidad del cu- rrículo, la correspondencia entre las intenciones de los docentes y sus estrategias, por citar algunas.

\section{NOTA}

Este trabajo forma parte de un proyecto financiado por el MCYT, con financiación parcial de fondos europeos FEDER, código BSO200204073-C02-02.

\section{REFERENCIAS BIBLIOGRÁFICAS}

BLOOME,D.,PURO,P.y THEODOROU,E. (1989). Procedural display and classroom lessons. Curriculum Inquiry, 19, pp. 265-291.

BROWN, A.L. (1992). Design Experiments: theoretical and Methodological Challenges in creating complex interventions in classroom settings. The Journal of the learning Sciences, 2, pp. 141-178.

CAZDEN, C. (1991). El discurso en el aula. El lenguaje de la enseñanza y el aprendizaje. Barcelona: Paidós-MEC.

DÍAZ DE BUSTAMANTE, J. (1999). «Problemas de aprendizaje en la interpretación de observaciones de estructuras biológicas con el microscopio.» Tesis doctoral. Universidade de Santiago de Compostela.

DÍAZ DE BUSTAMANTE, J. y JIMÉNEZ ALEIXANDRE, M.P. (2001). Communication in the laboratory sessions and sequences of arguments. Proceedings III Conference of European Researchers in Didactic of Biology (ERIDOB), Santiago de Compostela, pp. 247-260.

DRIVER, R., NEWTON, P. y OSBORNE, J. (2000). Establishing the norms of scientific argumentation in classrooms. Science Education. 84, pp. 287-312.

DUSCHL, R. (1997). Renovar la enseñanza de las ciencias. La importancia de las teorías y su desarrollo. Madrid: Narcea. Trad. de Restructuring Science Education (1990).

DUSCHL, R.A. y ERDURAN, S. (1996). Modelling the growth of scientific knowledge, en Weldorf, G., Osborne, J. y Scott, P. Research in Science Education in Europe. Londres: Falmer Press.

DUSCHL, R.A., ELLENBOGEN, K. y ERDURAN, S. (1999). Middle School Science students' dialogic argumentation. Comunicación en ESERA Conference. Kiel.

GEERTZ, C. (1987). La interpretación de las culturas. Barcelona: Gedisa.
HABERMAS, J. (1987). Teoría de la acción comunicativa. Madrid: Taurus.

HENNESSEY, G. (1991). «Analysis of concept change and status change in sixth graders' concepts of force and motion». Tesis doctoral. University of Wisconsin.

HINTIKKA, J. (1999). Inquiry as Inquiry: A logic of scientific discovery. Dordrecht: Kluwer Academic Publishers.

JIMÉNEZ ALEIXANDRE, M.P. (1998). Diseño curricular: indagación y razonamiento con el lenguaje de las ciencias. Enseñanza de las Ciencias, 16 (2), pp. 203-216.

JIMÉNEZ ALEIXANDRE, M.P. (2003). Comunicación y lenguaje en la clase de ciencias, en Jiménez (coord.), Caamaño, Oñorbe, Pedrinaci, De Pro. Enseñar ciencias. Barcelona: Graó.

JIMÉNEZ ALEIXANDRE, M.P., BUGALLO RODRÍGUEZ, A. y DUSCHL, R.A. (2000). «Doing the lesson» or «Doing Science»: Argument in High School Genetics. Science Education, 84, pp. 757-792.

JIMÉNEZ ALEIXANDRE, M.P., DÍAZ DE BUSTAMANTE, J. y DUSCHL, R.A. (1998). Scientific culture and School culture. Epistemic and procedural components. Comunicación en el congreso de NARST. San Diego, CA.

JIMÉNEZ ALEIXANDRE, M.P. y LÓPEZ RODRÍGUEZ, R. (2001). Designing a field code: environmental values in Primary school. Environmental Education Research, 7 (1), pp. 5-22.

JIMÉNEZ ALEIXANDRE, M.P., PEREIRO MUÑOZ, C. y AZNAR CUADRADO, V. (2000). Reasoning on environmental issues: an empirical study about environmental management in the 11th Grade, en Bayrhuber, H. y Mayer, J. (eds.). Empirical research on Environmental Education in Europe. Münster: Waxmann.

KELLY, G.J. y BROWN, C. (2000). Communicative demands 
of learning science through technological design: third grade students' construction of solar energy devices. Comunicación presentada en el congreso de AERA. New Orleans.

KELLY, G.J. y CRAWFORD, T. (1997). An ethnographic investigation of the discourse processes of school science. Science Education, 81, pp. 533-559.

KELLY, G.J., DRUCKER, S. y CHEN, K. (1998). Students' reasoning about electricity: combining performance assessment with argumentation analysis. International Journal of Science Education, 20, pp. 849-871.

KUHN, D. (1992). Thinking as Argument. Harvard Educational Review, 62, pp. 155-178.

KUHN, D. (1993). Science as argument: Implications for teaching and learning Scientific thinking. Science Education, 77 (3), pp. 319-337.

LATOUR, B. y WOOLGAR, S. (1995). La vida en el laboratorio. La construcción de los hechos científicos. Madrid: Alianza Universidad. Trad. Laboratory Life. The construction of scientific facts. Princeton University Press (1986).

LÓPEZ RODRÍGUEZ, R. (2001). «La integración de la educación ambiental en el diseño curricular: un estudio longitudinal en educación primaria». Tesis doctoral. Universidade de Santiago de Compostela.

McGINN, M. y ROTH W.M. (1999). Preparing Students for competent scientific practice: Implications of recent research in Science and Technology Studies. Educational Researcher, 28 (3), pp. 14-24.

OGBORN, J., KRESS, G., MARTINS, I. y McGILLICUDDY, K. (1998). Formas de explicar. La enseñanza de las ciencias en secundaria. Madrid: Santillana. Trad. de Explaining science in the classroom (1996).

PEREIRO MUÑOZ, C. (2001). «Desenvolvemento da capacidade de elaborar argumentos sobre impacto ambiental no contexto da aprendizaxe da Bioloxía e Xeoloxía no Bacharelato». Tesis doctoral. Universidade de Santiago de Compostela.

PONTECORVO, C. y GIRARDET, H. (1993). Arguing and reasoning in understanding Historical Topics. Cognition and Instruction, 11 (3-4), pp. 365-395.
REIGOSA CASTRO, C. y JIMÉNEZ ALEIXANDRE, M.P. (2000). La cultura científica en la resolución de problemas en el laboratorio. Enseñanza de las Ciencias, 18 (2), pp. 275-284.

REIGOSA CASTRO, C. (2002). «Discurso en el laboratorio durante la resolución de problemas de física y química: acciones, justificaciones, cultura científica y mediación». Tesis doctoral. Universidade de Santiago de Compostela.

REIGOSA CASTRO, C. y JIMÉNEZ ALEIXANDRE, M.P. (2001). Collaborative work in the Vygotskian ZPD: a case study in the Chemistry laboratory. Comunicación presentada en el congreso de ESERA. Tesalónica.

REIGOSA CASTRO, C., JIMÉNEZ ALEIXANDRE, M.P. y GARCÍA-RODEJA, E. (2001). Acciones y discurso durante la realización de un problema de física: referentes en la justificación de las propuestas. Comunicación presentada en el congreso de Enseñanza de las Ciencias.

SARDÁ JORGE, A. y SANMARTÍ PUIG, N. (2000). Enseñar a argumentar científicamente: un reto de las clases de ciencias. Enseñanza de las Ciencias, 18 (3), pp. 405-422.

SUTTON, C. (1992). Words, Science and Learning. Buckingham: Open University Press.

SUTTON, C. (1997). Ideas sobre la ciencia e ideas sobre el lenguaje. Alambique, 12, pp. 8-32.

TOULMIN, S. (1958). The uses of argument. Nueva York: Cambridge University Press.

VYGOTSKI, L.S. (1979). El desarrollo de los procesos psicológicos superiores. Barcelona: Crítica.

WALTON, D. (1989). Informal Logic. A Handbook for critical argumentation. Cambridge: Cambridge University Press.

WALTON, D. (1996). Argumentation schemes for presumptive reasoning. Mahwah, N.J.: Lawrence Erlbaum.

ZOHAR, A. y NEMET, F. (2002). Fostering Students' Knowledge and Argumentation Skills Through Dilemmas in Human Genetics. Journal of Research in Science Teaching, 39 , pp. 35-62. 


\section{APÉNDICE}

Las huellas del ladrón

Nombre

Muestra núm.

\section{Las huellas del ladrón}

En el laboratorio del centro fue robada la cámara de vídeo. Sin embargo, el ladrón se lastimó, dejando unos restos alguna parte de su cuerpo en la puerta del armario. Con estos pequeños restos se han hecho preparaciones que han sido inspeccionados en el microscopio.

Debéis investigar a qué sospechoso pertenece, justificando vuestras conclusiones.

Ésta es la lista de los sospechosos, que proceden de una colonia de seres extraterrestres, por lo que pueden ser algo distintos de los animales y vegetales que se encuentran en la Tierra.

A.1. CLOROFILIO: Las células de este individuo son como las de los vegetales terrestres. Recibe este nombre porque posee clorofila (en los cloroplastos); su nutrición es, pues, fotosintética. Además puede presentar estomas por donde realiza el intercambio gaseoso.

A.2. TUNELIO: Las células de este individuo son como las de los vegetales terrestres. Recibe este nombre porque vive bajo tierra, carece, pues, de clorofila. Los núcleos de sus células son bastante visibles. Sin embargo, no presentan estomas.

B.1. GALIÑNLIO: Las células de este individuo son como las de los animales terrestres. Recibe este nombre por ser volador. Tiene sangre roja (con hemoglobina), en la que son visibles más de un tipo de células.

B.2. ARAÑILIO: Las células de este individuo son como las de los animales terrestres. Respira a través de la piel y no tiene sangre. Sus células, de forma irregular, se encuentran distribuidas en capas, y son todas del mismo tipo. 